\title{
Chiral Symmetry Breaking and the Soliton-Stripe Pattern in Langmuir Monolayers and Smectic Films *†
}

\author{
Xiaofeng Ren \\ Department of Mathematics and Statistics \\ Utah State University \\ Logan, UT 84322-3900, USA
}

\author{
Juncheng Wei ${ }^{\ddagger}$ \\ Department of Mathematics \\ Chinese University of Hong Kong \\ Shatin, Hong Kong
}

May 21, 2004

\begin{abstract}
Chiral symmetry breaking in Langmuir monolayers and smectic films may be modelled by a system of two coupled fields: A scalar field which represents the chirality of the molecules and a vector field which represents the tilt direction of the molecules. In a particular parameter range we prove the existence of a soliton-stripe pattern, using the $\Gamma$-limit theory in perturbative variational calculus. This pattern, modelled by one-dimensional local minimizers of the free energy of the system, consists of stripes of molecules with distinct chirality in the film delineated by sharp domain walls.
\end{abstract}

PACS codes. 64.60.Fr, 68.55.Jk, 02.30.Xx

2000 Mathematics Subject Classification. 49J99, 82D99

Key words. Langmuir monolayer, smectic film, soliton-stripe pattern, local minimizer, Гconvergence.

\section{Introduction}

There is a close relation between molecular chirality and pattern formation in liquid crystals. In bulk three-dimensional systems chiral molecules can form a cholesteric phase with a helical pattern of twist in the molecular director, and a smectic- $C^{*}$ phase, in which the director rotates from layer to layer, De Gennes and Prost [7].

In two-dimensional systems chiral molecules can form a striped pattern of parallel defect walls. More interestingly in some Langmuir monolayers and freely suspended smectic films of nonchiral molecules similar striped patterns can occur. In these systems chiral symmetry is spontaneously broken leading to a chiral phase composed of nonchiral molecules. We study this phenomenon in this paper using a model proposed by Selinger, Wang, Bruinsma and Knobler [27].

*Abbreviated title. Molecular Chirality.

${ }^{\dagger}$ Corresponding author: Juncheng Wei, Phone: 852 2609-7967, Fax: 852 2603-5154, E-mail: wei@math.cuhk.edu.hk

¥Supported in part by a Direct Grant from CUHK and an Earmarked Grant of RGC of Hong Kong. 


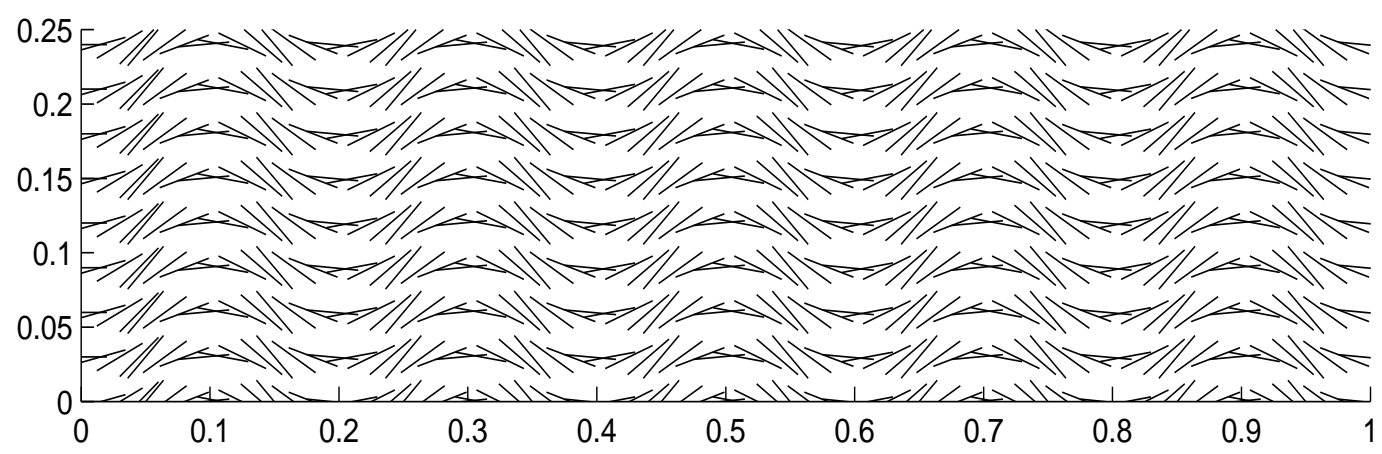

Figure 1: A soliton-stripe pattern of a chiral liquid crystal film. The director field is drawn on the two dimensional film. Slope increases on lamellar stripes of one chiral type and decreases on stripes of the other type.

There are several mechanisms that can cause chiral symmetry breaking in two-dimensions. We summarize them here. The reader should consult [27] for more detail and references. In a Langmuir monolayer, if it is in a tilted hexatic phase, the tilt direction can be locked at an angle between $0^{\circ}$ and $30^{\circ}$ from one of the local bond directions. This relation between tilt order and bond-orientational order breaks chiral symmetry. The chiral order parameter would be $u=\sin (6(\phi-\theta))$, where $\phi$ is the tilt azimuth and $\theta$ the bond orientation. Second even if the monolayer is not in a tilted hexatic phase, the molecules might pack on the $2 \mathrm{D}$ surface in two inequivalent ways that are mirror images of each other. The chiral order parameter would be the difference in the densities of the two packings. Third if the monolayer is composed of a racemic mixture of two opposite enantiomers, the racemic mixture can separate to form chiral domains. In that case the chiral order parameter would be the differences in densities of the two enantiomers. In a freely suspended smectic thin film where the top and bottom surfaces are equivalent, $\mathbf{n}$ is equivalent to $-\mathbf{n}$. A tilted hexatic film of cylindrical molecules always has an inversion symmetry. Hence $\sin (6(\phi-\theta))$ is not a chiral order parameter. But chiral symmetry can still be broken by the other two mechanisms described above.

Like the Landau-de Gennes [6] model for three-dimensional smectics A, the model in [27] also has two order parameters, although its mathematical structure is simpler. The first is a scalar field $u$ that characterizes the chirality of the molecules. $u>0$ indicates one chiral state and $u<0$ indicates the other chiral sate. The second is a two-dimensional vector field $\mathbf{c}$ of unit length, which characterizes the director of the molecules. It is actually the normalized projection of the 3D molecular director $\mathbf{n}$ into the plane of the film. We neglect variations in the magnitude of the tilt. Suppose the film occupies $\Omega \subset \mathbf{R}^{2}$, so for $r \in \Omega, u=u(r) \in \mathbf{R}$ and $\mathbf{c}=\mathbf{c}(r) \in S^{1} \subset \mathbf{R}^{2}$, where $S^{1}$ is the unit circle in $\mathbf{R}^{2}$. The free energy of the system is

$$
\mathcal{F}(u, \mathbf{c})=\int_{\Omega}\left(\frac{\kappa}{2}|\nabla u|^{2}-\frac{t}{2} u^{2}+\frac{s}{4} u^{4}+\frac{K_{1}}{2}(\nabla \cdot \mathbf{c})^{2}+\frac{K_{3}}{2}(\nabla \times \mathbf{c})^{2}-\lambda u \nabla \times \mathbf{c}\right) d r .
$$

In (1.1) $\kappa, t, s, K_{1}, K_{3}, \lambda$ are all positive constants.

We make the single-Frank-constant approximation $K_{1}=K_{3}=\tilde{K}$. When c can be expressed in 
the polar coordinates globally, i.e. $\mathbf{c}=(\cos v, \sin v)$, we simplify (1.1) to

$$
\mathcal{F}(u, v)=\int_{\Omega}\left(\frac{\kappa}{2}|\nabla u|^{2}-\frac{t}{2} u^{2}+\frac{s}{4} u^{4}+\frac{\tilde{K}}{2}|\nabla v|^{2}-\lambda u\left(\frac{\partial \sin v}{\partial r_{1}}-\frac{\partial \cos v}{\partial r_{2}}\right)\right) d r .
$$

We assume that $\Omega$ is a square: $\Omega=(0, L) \times(0, L)$. To separate the domain effect we scale $\Omega$ to $D=(0,1) \times(0,1)$ by introducing $x=\left(x_{1}, x_{2}\right) \in D$ so that $r=L x$. We scale $u, \mathbf{c}$ and $v$ to

$$
\psi(x)=\frac{\sqrt{s}}{2 \sqrt{t}} u(r), \quad \phi(x)=v(r),
$$

so that

$$
\mathcal{F}(u, v)=\frac{4 L^{2} t^{2}}{s} F(\psi, \phi)-\frac{L^{2} t^{2}}{4 s},
$$

where $F(\psi, \phi)$, the rescaled dimensionless free energy, is

$$
F(\psi, \phi)=\int_{D}\left(\frac{\epsilon^{2}}{2}|\nabla \psi|^{2}+W(\psi)+\frac{\epsilon \gamma}{2}|\nabla \phi|^{2}-\epsilon \gamma \beta \psi\left(\frac{\partial \sin \phi}{\partial x_{1}}-\frac{\partial \cos \phi}{\partial x_{2}}\right)\right) d x,
$$

The new parameters in (1.5) are related to the old parameters in (1.1) via

$$
\epsilon^{2}=\frac{\kappa}{L^{2} t}, \epsilon \gamma=\frac{\tilde{K} s}{8 L^{2} t^{2}}, \epsilon \gamma \beta=\frac{\lambda \sqrt{s}}{2 L \sqrt{t^{3}}} .
$$

The function $W$ in $(1.5)$ is

$$
W(\psi)=\left(\psi^{2}-1 / 4\right)^{2} .
$$

The soliton-stripe pattern [27] is a lamellar pattern of $\psi$ and $\phi$ that only vary in one direction. In the context of a chiral liquid crystal the chiral field $\psi$ takes values close to $-1 / 2$ in one lamellar stripe. Next to it is another lamellar stripe where $\psi$ is close to $1 / 2$. The film is covered by a large number of lamellar stripes of alternating types. The stripes are of finite width and are delineated by sharp domain walls (solitons). ${ }^{1}$ The director field $\phi$ also varies along these stripes. When $\psi$ is close to $1 / 2, \phi$ increases continuously in $(-\pi / 2, \pi / 2)$, and when $\psi$ is close to $-1 / 2, \phi$ decreases continuously in $(-\pi / 2, \pi / 2)$. Figure 1 shows the director field $\phi$ on a film, and Figure 2 shows how $\psi$ and $\phi$ vary (in their asymptotic limit) along the direction perpendicular to the stripes.

In this paper we will find soliton-stripe solutions in the parameter range

$$
0<\epsilon \ll 1, \gamma \sim 1, \beta \sim 1 .
$$

In terms of the original parameters we assume

$$
\frac{1}{L} \sqrt{\frac{\kappa}{t}} \ll 1, \frac{\tilde{K} s}{L \sqrt{\kappa t^{3}}} \sim 1, \frac{L \lambda}{\tilde{K}} \sqrt{\frac{t}{s}} \sim 1 .
$$

One may think (1.9) as a condition under which a separation of two scales occur. The two scales are the thickness of the interfaces (solitons) and the width of the stripes. According to (1.9) if the parameters $t, s, \kappa, \tilde{K}$ and $\lambda$ satisfy

$$
\frac{\kappa t}{\tilde{K} s} \ll 1, \frac{\lambda}{t} \sqrt{\frac{s}{k}} \sim 1
$$

\footnotetext{
${ }^{1}$ There is another lamellar pattern, the sinusoidal pattern, where domain walls are not sharp [27].
} 
we may choose

$$
L \sim \frac{\tilde{K} s}{\sqrt{\kappa t^{3}}}
$$

Then the sample size is of the same order as the stripe width while the interface thickness is of smaller order.

Since a soliton-stripe pattern varies along one direction, we assume that $(\psi, \phi)$ depends on $x_{1} \in(0,1)$ only, which we denote by $x$ throughout the rest of the paper. To eliminate unnecessary boundary effects we identify the boundary points 0 and 1 to impose the periodic boundary condition. The interval $(0,1)$ is now $\mathbf{R} / \mathbf{Z}$. And (1.5) becomes

$$
F_{\epsilon}(\psi, \phi)=\int_{0}^{1}\left(\frac{\epsilon^{2}}{2}\left(\psi^{\prime}\right)^{2}+W(\psi)+\frac{\epsilon \gamma}{2}\left(\phi^{\prime}\right)^{2}+\epsilon \gamma \beta \psi^{\prime} \sin \phi\right) d x
$$

defined in the admissible set $W^{1,2}(\mathbf{R} / \mathbf{Z}) \times W^{1,2}(\mathbf{R} / \mathbf{Z})$. Because $\mathbf{R} / \mathbf{Z}$ acts on $\mathbf{R} / \mathbf{Z}$ as a translation group, we will often encounter degeneracy caused by this action. We will frequently use phrases like 'up to translation' or 'modulo translation'. Also note that if we let $c(x)=\mathbf{c}(r)$, then in one dimension here $c$ is a map from $\mathbf{R} / \mathbf{Z} \equiv S^{1}$ to $S^{1}$. The assumed existence of a globally continuous $\phi$ so that $c=(\cos \phi, \sin \phi)$ is equivalent to the condition that the winding number of $c$ is zero.

In view of (1.8) we hold $\gamma$ and $\beta$ fixed and treat $\epsilon$ as a small parameter, so (1.12) is a singularly perturbed variational problem. The Euler-Lagrange equations of (1.12) are

$$
\begin{aligned}
-\epsilon^{2} \psi^{\prime \prime}+W^{\prime}(\psi)-\epsilon \gamma \beta(\sin \phi)^{\prime} & =0 \\
-\phi^{\prime \prime}+\beta \psi^{\prime} \cos \phi & =0
\end{aligned}
$$

with the periodic boundary condition. The main result of this paper is the following existence theorem.

Theorem 1.1 For each positive even integer $K$ the functional $F_{\epsilon}$ has a local minimizer $\left(\psi_{\epsilon}, \phi_{\epsilon}\right)$ when $\epsilon$ is sufficiently small. It satisfies the Euler-Lagrange equations (1.13) and (1.14) and has the properties $\lim _{\epsilon \rightarrow 0}\left\|\psi_{\epsilon}-\psi^{0}\right\|_{2}=0, \lim _{\epsilon \rightarrow 0}\left\|\phi_{\epsilon}-\phi^{0}\right\|_{1,2}=0$ modulo translation and $\lim _{\epsilon \rightarrow 0} \epsilon^{-1} F_{\epsilon}\left(\psi_{\epsilon}, \phi_{\epsilon}\right)=$ $J\left(\psi^{0}\right)$.

$\|\cdot\|_{2}$ denotes the $L^{2}$-norm, and $\|\cdot\|_{1,2}$ denotes the $W^{1,2}$-norm. $J$ is defined in $(2.20)$. That $\psi_{\epsilon}$ develops a Soliton-Stripe pattern of $K$ domain walls as $\epsilon \rightarrow 0$ lies in the fact that the limiting profile $\psi_{0}$ of $\psi_{\epsilon}$ is a step function with $K$ jump points of equal distance:

$$
\psi^{0}(x)=\left\{\begin{aligned}
1 / 2 & \text { on }(0,1 /(2 K)) \\
-1 / 2 & \text { on }(1 /(2 K), 3 /(2 K)) \\
1 / 2 & \text { on }(3 /(2 K), 5 /(2 K)) \\
-1 / 2 & \text { on }(5 /(2 K), 7 /(2 K)) \\
\ldots & \\
-1 / 2 & \text { on }((2 K-3) /(2 K),(2 K-1) /(2 K)) \\
1 / 2 & \text { on }((2 K-1) /(2 K), 1)
\end{aligned}\right.
$$




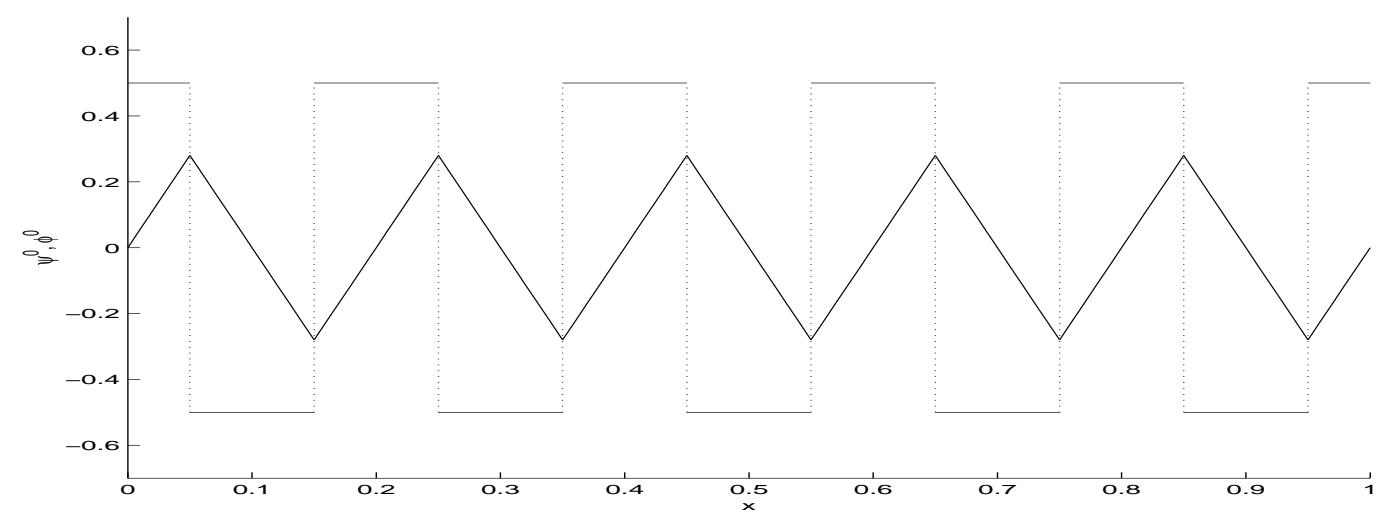

Figure 2: The discontinuous step function is $\psi^{0}$ and the zigzag function is $\phi^{0}$.

The corresponding $\phi^{0}$ is

$$
\phi^{0}(x)=\left\{\begin{aligned}
2 K \xi x & \text { on }(0,1 /(2 K)) \\
-2 K \xi(x-1 / K) & \text { on }(1 /(2 K), 3 /(2 K)), \\
2 K \xi(x-2 / K) & \text { on }(3 /(2 K), 5 /(2 K)), \\
-2 K \xi(x-3 / K) & \text { on }(5 /(2 K), 7 /(2 K)), \\
\cdots & \text { on }((2 K-3) /(2 K),(2 K-1) /(2 K)), \\
-2 K \xi(x-(K-1) / K) & \text { on }((2 K-1) /(2 K), 1) \\
2 K \xi(x-1) &
\end{aligned}\right.
$$

Here $\xi$ is the unique solution of

$$
\cos \xi=\frac{4 K}{\beta} \xi
$$

in $(0, \pi / 2)$. See Figure 2. Note that $\phi^{0}$ satisfies $(1.14)$ when $\psi=\psi^{0}$.

Mathematical studies on periodic patterns with sharp domain walls started rather recently. Many works have been done to the block copolymer problem. The literature there includes Nishiura and Ohnishi [15], Ohnishi et al [16], Ren and Wei [18, 20, 19, 22, 23, 21, 26], Choksi [3], Fife and Hilhorst [10], Henry [11], and Choksi and Ren [4]. Ren and Wei [24] deals with bending membranes, and [25] studies charged monolayers. Also see Ren and Truskinovsky [17], and Chmaj and Ren [2].

\section{The $\Gamma$-limit}

The $\Gamma$-limit theory of De Giorgi [8] is a rigorous singular perturbation theory in the calculus of variations. An introduction to the theory may be found in Dal Maso [5]. In this theory there is a perturbed variational problem, which is often a standard one with a small parameter, say $\epsilon$. The Euler-Lagrange equation is a differential equation. The limiting problem, as $\epsilon \rightarrow 0$, is usually a geometric problem, whose extremals solve a free boundary problem. Certain properties of the limiting problem are carried over to the perturbed problem (Corollary 2.4). In this sense the perturbed problem is reduced to the limiting problem. 
Different from our earlier works based on the $\Gamma$-convergence theory [18, 19, 23, 24, 25], here we build a local version of the theory near $\psi^{0}$ and $\phi^{0}$.

Lemma $2.1 \phi^{0}$ is a stable solution of (1.14) where $\psi=\psi^{0}$ in the sense that the eigenvalues of the linearized operator of (1.14) at $\phi^{0}$ are all positive.

Proof. The eigenvalue problem at $\phi^{0}$ is

$$
-q^{\prime \prime}-\beta\left(\psi^{0}\right)^{\prime}\left(\sin \phi^{0}\right) q=\lambda q .
$$

Note that $\left(\psi^{0}\right)^{\prime}=\sum_{j=1}^{K}(-1)^{j} \delta\left(\cdot-x_{j}^{0}\right)$ and $\sin \left(\phi^{0}\left(x_{j}^{0}\right)\right)=(-1)^{j+1} \sin \xi$. Hence (2.1) is simplified to

$$
-q^{\prime \prime}+\beta \sin \xi\left(\sum_{j=1}^{K} \delta\left(\cdot-x_{j}^{0}\right)\right) q=\lambda q .
$$

Multiplying (2.2) by $q$ and integrating by parts, we obtain

$$
\int_{0}^{1}\left(q^{\prime}\right)^{2} d x+\beta \sin \xi \sum_{j=1}^{K}\left(q\left(x_{j}\right)\right)^{2}=\lambda \int_{0}^{1} q^{2} d x .
$$

On the other hand

$$
\int_{0}^{1} q^{2} d x \leq 2\left(q\left(x_{1}\right)\right)^{2}+2 \int_{0}^{1}\left(q^{\prime}\right)^{2} d x .
$$

(2.3) and (2.4) give a positive lower bound for $\lambda$ :

$$
\lambda \geq \frac{1}{2} \min \{1, \beta \sin \xi\}
$$

from which the lemma follows.

Lemma 2.2 There is an $L^{2}$ neighborhood of $\psi^{0}$ such that for every $\psi$ in the neighborhood there is a solution $\phi$ of (1.14). The $(\psi, \phi)$ pairs form a smooth curve under the $L^{2} \times W^{1,2}$ norm which passes through $\left(\psi^{0}, \phi^{0}\right)$. Furthermore the eigenvalues of the linearized operator of (1.14) at every $\phi$ are all positive.

Proof. We define $Q: L^{2}(\mathbf{R} / \mathbf{Z}) \times W^{1,2}(\mathbf{R} / \mathbf{Z}) \rightarrow W^{1,2}(\mathbf{R} / \mathbf{Z})$ by

$$
Q(\psi, \phi)=\phi+\left(-\frac{d^{2}}{d x^{2}}+\beta\right)^{-1}\left(-\beta \phi+\beta \psi^{\prime} \cos \phi\right)
$$

where $\left(-\frac{d^{2}}{d x^{2}}+\beta\right)^{-1}$ is from $W^{-1,2}(\mathbf{R} / \mathbf{Z})$ to $W^{1,2}(\mathbf{R} / \mathbf{Z})$. Then (1.14) is equivalent to

$$
Q(\psi, \phi)=0 .
$$

Lemma 2.1 implies that the Fréchet derivative

$$
D_{\phi} Q\left(\psi^{0}, \phi^{0}\right): W^{1,2}(\mathbf{R} / \mathbf{Z}) \rightarrow W^{1,2}(\mathbf{R} / \mathbf{Z})
$$


is a bounded bijective linear operator. By the Implicit Function Theorem the solutions of (2.7) near $\left(\psi^{0}, \phi^{0}\right)$ form a smooth curve which passes through $\left(\psi^{0}, \phi^{0}\right)$.

As in Lemma 2.1 solutions from this curve continue to be stable. To see this consider the eigenvalue problem at $\phi$ :

$$
-q^{\prime \prime}-\beta \psi^{\prime}(\sin \phi) q=\lambda q
$$

Multiplying (2.8) by $q$ and integrating over $(0,1)$, we obtain

$$
\int_{0}^{1}\left(\left(q^{\prime}\right)^{2}-\beta \psi^{\prime}(\sin \phi) q^{2}\right) d x=\lambda \int_{0}^{1} q^{2} d x .
$$

The integral in the right side is estimated as in (2.4). The left side is written as

$\int_{0}^{1}\left(\left(q^{\prime}\right)^{2}-\beta \psi^{\prime}(\sin \phi) q^{2}\right) d x=\int_{0}^{1}\left(\left(q^{\prime}\right)^{2}-\beta\left(\psi^{0}\right)^{\prime}\left(\sin \phi^{0}\right) q^{2}\right) d x+\beta \int_{0}^{1}\left(\left(\psi^{0}\right)^{\prime}\left(\sin \phi^{0}\right) q^{2}-\psi^{\prime}(\sin \phi) q^{2}\right) d x$.

The first integral in (2.10) is estimated as in Lemma 2.1, i.e.

$$
\int_{0}^{1}\left(\left(q^{\prime}\right)^{2}-\beta\left(\psi^{0}\right)^{\prime}\left(\sin \phi^{0}\right) q^{2}\right) d x \geq \min \{1, \beta \sin \xi\}\left(\left(q\left(x_{1}^{0}\right)^{2}+\int_{0}^{1}\left(q^{\prime}\right)^{2} d x\right) .\right.
$$

The second integral in (2.10) is estimated as follows

$$
\begin{aligned}
& \left|\int_{0}^{1}\left(\left(\psi^{0}\right)^{\prime}\left(\sin \phi^{0}\right) q^{2}-\psi^{\prime}(\sin \phi) q^{2}\right) d x\right| \\
& \leq \quad \int_{0}^{1}\left|-\psi^{0}\left(\cos \phi^{0}\right)\left(\phi^{0}\right)^{\prime}+\psi(\cos \phi) \phi^{\prime}\right| q^{2} d x \\
& \quad+\int_{0}^{1}\left|-2 \psi^{0}\left(\sin \phi^{0}\right) q q^{\prime}+\psi(\sin \phi) q q^{\prime}\right| d x \\
& \quad \leq \quad C\left(\left\|\psi^{0}-\psi\right\|_{2}^{2}+\left\|\phi^{0}-\phi\right\|_{1,2}^{2}\right)^{1 / 2}\left(\left(q\left(x_{1}^{0}\right)^{2}+\int_{0}^{1}\left(q^{\prime}\right)^{2} d x\right) .\right.
\end{aligned}
$$

(2.4), (2.9), (2.10), (2.11) and (2.12) yield a positive lower bound for $\lambda$ if $(\psi, \phi)$ is close to $\left(\psi^{0}, \phi^{0}\right)$ enough.

We define

$$
X=\left\{\psi \in L^{2}(\mathbf{R} / \mathbf{Z}):\left\|\psi-\psi^{0}(\cdot-y)\right\|_{2} \leq \delta \text {, for some } y \in \mathbf{R} / \mathbf{Z}\right\} .
$$

In (2.13) $\delta$ is small enough so that every $\psi \in X$ with $\left\|\psi-\psi^{0}\right\| \leq \delta$ is in the neighborhood of $\psi^{0}$ of Lemma 2.2. For each $\psi$ there denote the corresponding solution $\phi$ of (1.14) constructed in Lemma 2.2 by $\phi_{\psi}$. Set

$$
E(\psi)=\int_{0}^{1}\left(\frac{1}{2}\left(\phi_{\psi}^{\prime}\right)^{2}+\beta \psi^{\prime} \sin \phi_{\psi}\right) d x, \quad \psi \in X
$$

Define, in $W^{1,2}(\mathbf{R} / \mathbf{Z}) \cap X$,

$$
I_{\epsilon}(\psi)=\int_{0}^{1}\left(\frac{\epsilon^{2}}{2}\left(\psi^{\prime}\right)^{2}+W(\psi)\right) d x+\epsilon \gamma E(\psi) .
$$


Note that

$$
I_{\epsilon}(\psi)=F_{\epsilon}\left(\psi, \phi_{\psi}\right) .
$$

Hence to prove Theorem 1.1 we look for a local minimizer $\psi$ of $I_{\epsilon}$ in the interior of $X$, because by Lemma $2.2\left(\psi, \phi_{\psi}\right)$ would be a local minimizer of $F_{\epsilon}$. To this end we apply the $\Gamma$-convergence theory to $I_{\epsilon}$.

For technical reasons we extend the domain of $I_{\epsilon}$ to $X$ by setting $I(\psi)=\infty$ if $\psi \notin W^{1,2}(\mathbf{R} / \mathbf{Z})$. The singular limit (the $\Gamma$-limit) of $\epsilon^{-1} I_{\epsilon}$, denoted by $J$ in this paper, is a variational problem initially defined in

$$
B V(\mathbf{R} / \mathbf{Z},\{ \pm 1 / 2\}) \cap X
$$

$B V(\mathbf{R} / \mathbf{Z},\{ \pm 1 / 2\})$ is the class of periodic functions of bounded variation whose values are $-1 / 2$ or $1 / 2$. Each function there has a finite number of jumps between $-1 / 2$ and $1 / 2$. A more formal description of these functions may be found in Evans and Gariepy [9, chapter 5]. Naturally for each positive, even integer $N$ we set

$$
A_{N}=\{\psi \in B V(\mathbf{R} / \mathbf{Z},\{ \pm 1 / 2\}): \psi \text { has } N \text { jumps }\}
$$

Then we have a decomposition

$$
B V(\mathbf{R} / \mathbf{Z},\{ \pm 1 / 2\})=\bigcup_{N=2, \text { even }}^{\infty} A_{N}
$$

For each $\psi$ in $B V(\mathbf{R} / \mathbf{Z},\{ \pm 1 / 2\}) \cap X$ we define

$$
J(\psi)=\tau N+\gamma E(\psi), \text { if } \psi \in A_{N} .
$$

$\tau$ is a positive constant defined by

$$
\tau=\int_{-1 / 2}^{1 / 2} \sqrt{2 W(u)} d u
$$

It is called the interface tension. Again we extend $J$ trivially to $X$ by taking $J(\psi)=\infty$ if $\psi \in$ $X \backslash B V(\mathbf{R} / \mathbf{Z},\{ \pm 1 / 2\})$.

Lemma 2.3 Let $X$ be equipped with the $L^{2}$ metric.

1. As $\epsilon \rightarrow 0, \epsilon^{-1} I_{\epsilon} \Gamma$-converges to $J$ in the following sense.

(a) For every family $\left\{\psi_{\epsilon}\right\} \subset X$ with $\lim _{e \rightarrow 0} \psi_{\epsilon}=\psi$, $\liminf _{\epsilon \rightarrow 0} \epsilon^{-1} I_{\epsilon}\left(\psi_{\epsilon}\right) \geq J(\psi)$;

(b) For every $\psi \in X$, there is a family $\left\{\psi_{\epsilon}\right\} \subset X$ such that $\lim _{\epsilon \rightarrow 0} \psi_{\epsilon}=\psi$ and $\limsup _{\epsilon \rightarrow 0} \epsilon^{-1} I_{\epsilon}\left(\psi_{\epsilon}\right) \leq$ $J(\psi)$

2. Let $\epsilon_{j}$ be a sequence of positive numbers converging to 0 , and $\left\{\psi_{j}\right\}$ a sequence in $X$. If $\epsilon_{j}^{-1} I_{\epsilon_{j}}\left(\psi_{j}\right)$ is bounded above in $j$, then $\left\{\psi_{j}\right\}$ is relatively compact in $X$ and its cluster points belong to $B V(\mathbf{R} / \mathbf{Z},\{ \pm 1 / 2\})$. 
Proof. We view $\epsilon^{-1} I_{\epsilon}$ as a sum of a local part

$$
H_{\epsilon}(\psi):=\int_{0}^{1}\left(\frac{\epsilon}{2}\left(\psi^{\prime}\right)^{2}+\frac{1}{\epsilon} W(\psi)\right) d x,
$$

and an $\epsilon$-independent, perturbative, nonlocal part $\gamma E$. Regarding $E$, we note that $\psi \rightarrow E(\psi)$ is continuous from $X$ to $\mathbf{R}$.

After making some minor modifications (change $L^{1}$ to $L^{2}$ ) in the proof of Propositions 1 and 2 of Modica [14], we find that $H_{\epsilon} \Gamma$-converges to $H_{0}$. Here

$$
H_{0}(\psi):=\tau N \text {, if } \psi \in A_{N} .
$$

Because $E: X \rightarrow R$ is a continuous functional, by the definition of $\Gamma$-convergence $\epsilon^{-1} I_{\epsilon}=H_{\epsilon}+\gamma E$ $\Gamma$-converges to $J=H_{0}+\gamma E$.

Statement 2 of the lemma is a kind of uniform coercivity property. The proof is similar to the one in $[17$, Appendix]

The next result proved by Kohn and Sternberg [12] asserts that as a corollary of Lemma 2.3 near every isolated local minimizer of $J$ there exists a local minimizer of $I_{\epsilon}$. The original result [12, Theorem 2.1] deals with a domain with boundary. Here on $\mathbf{R} / \mathbf{Z}$ we must take the translation invariance of $I_{\epsilon}$ into consideration and state the result a little differently. Define a manifold of translates of $\psi_{0}$

$$
M\left(\psi_{0}\right):=\left\{\psi \in X: \psi(\cdot)=\psi_{0}(\cdot-y), y \in \mathbf{R} / \mathbf{Z}\right\}
$$

and a tube like neighborhood of $M\left(\psi_{0}\right)$

$$
N_{\delta}\left(\psi_{0}\right):=\left\{\psi \in X:\left\|\psi(\cdot)-\psi_{0}(\cdot-y)\right\|<\delta, \text { for some } y \text { in } \mathbf{R} / \mathbf{Z}\right\} .
$$

Corollary 2.4 Let $\delta>0$ and $\psi_{0} \in X_{m}$ be such that $J\left(\psi_{0}\right)<J(\psi)$ for all $\psi \in N_{\delta}\left(\psi_{0}\right) \backslash M\left(\psi_{0}\right)$. Then there exist $\epsilon_{0}>0$ and $\psi_{\epsilon} \in N_{\delta / 2}\left(\psi_{0}\right)$ for all $\epsilon<\epsilon_{0}$ such that $I_{\epsilon}\left(\psi_{\epsilon}\right) \leq I_{\epsilon}(\psi)$ for all $\psi \in N_{\delta / 2}\left(\psi_{0}\right)$. In addition $\psi_{\epsilon} \rightarrow \psi_{0}$ up to translation.

Lemma 2.5 If $\psi$ strictly minimizes $J$ in $A_{K}$ locally, up to translation, then the corresponding $\psi$ is a strict local minimizer of $J$ in $X$, modulo translation.

Proof. Suppose that the conclusion is false. There would be a sequence of $\psi_{j}$ such that $\psi_{j} \neq \psi$ modulo translation, $\psi_{j} \rightarrow \psi$ and $J\left(\psi_{j}\right) \leq J(\psi)$. The $L^{2}$-continuity of $E \operatorname{implies~} \lim _{j \rightarrow \infty} E\left(\psi_{j}\right)=$ $E(\psi)$. Therefore

$$
\limsup _{j \rightarrow \infty} H_{0}\left(\psi_{j}\right) \leq H_{0}(\psi) .
$$

On the other hand the lower semicontinuity theorem of BV functions ([9], Theorem 1, p. 172) states

We deduce that

$$
\liminf _{j \rightarrow \infty} H_{0}\left(\psi_{j}\right) \geq H_{0}(\psi)
$$

$$
\lim _{j \rightarrow \infty} H_{0}\left(\psi_{j}\right)=H_{0}(\psi) .
$$

Hence for large $j, \psi_{j}$ has exactly $K$ jumps and is in $A_{K}$. But this is inconsistent with $\psi_{j} \rightarrow \psi$, $J\left(\psi_{j}\right) \leq J(\psi)$, and the assumption of the lemma.

In $A_{K} H_{0}$ is constant. So minimizing $J$ means minimizing $E$. Now the study of $J$ in $X$ is reduced to the study of $E$ in $A_{K} \cap X$. Because of Lemma 2.2, Corollary 2.4 and Lemma 2.5, to prove Theorem 1.1 we need to show that $\psi^{0}$ is a strict local minimizer of $E$ in $A_{K} \cap X$ up to translation. 


\section{$3 \psi^{0}$ as a local minimizer of $E$}

When $\psi$ is in $A_{K} \cap X$, it is a step function determined by the jumps in $\mathbf{R} / \mathbf{Z}$ which we denote by $x_{1}, x_{2}, \ldots, x_{K}$, namely

$$
\psi(x)=\left\{\begin{aligned}
1 / 2 & \text { if } x \in\left(0, x_{1}\right) \\
-1 / 2 & \text { if } x \in\left(x_{1}, x_{2}\right) \\
1 / 2 & \text { if } x \in\left(x_{2}, x_{3}\right) \\
\ldots & \\
-1 / 2 & \text { if } x \in\left(x_{K-1}, x_{K}\right) \\
1 / 2 & \text { if } x \in\left(x_{K}, 1\right)
\end{aligned}\right.
$$

and

$$
\psi^{\prime}=\sum_{j=1}^{K}(-1)^{j} \delta\left(\cdot-x_{j}\right)
$$

Let

$$
g(z)=\frac{z^{2}}{2}-\frac{z}{2}+\frac{1}{12} \text { in }(0,1), \text { and periodically extended to } \mathbf{R} .
$$

Then the Green function of $-\frac{d^{2}}{d x^{2}}$ with the periodic boundary condition is $g(x-y)$. This allows us to rewrite (1.14) as

$$
\phi(x)-\bar{\phi}=\beta \sum_{j=1}^{K}(-1)^{j+1} g\left(x-x_{j}\right) \cos \phi_{j}
$$

for the corresponding $\phi_{\psi}$. We write this $\phi_{\psi}$ as $\phi$ for simplicity. Here $\bar{\phi}=\int_{0}^{1} \phi(x) d x$ is the average of $\phi$. We have also introduced the abbreviation $\phi_{j}=\phi\left(x_{j}\right)$. By integrating (1.14) we find

$$
\sum_{j=1}^{K}(-1)^{j} \cos \phi_{j}=0
$$

Because, by (1.14), (3.5) and (3.4),

$$
\begin{aligned}
\int_{0}^{1}\left(\phi^{\prime}\right)^{2} d x & =-\beta \int_{0}^{1} \psi^{\prime} \phi \cos \phi d x=-\beta \int_{0}^{1} \psi^{\prime} \cos \phi\left(\bar{\phi}+\beta \sum_{j=1}^{K}(-1)^{j+1} g\left(x-x_{j}\right)\right) d x \\
& =\beta^{2} \sum_{i, j=1}^{K}(-1)^{i+j} g\left(x_{i}-x_{j}\right) \cos \phi_{i} \cos \phi_{j}
\end{aligned}
$$

we can now rewrite $(2.14)$ as

$$
E\left(x_{1}, x_{2}, \ldots, x_{K}\right)=\frac{\beta^{2}}{2} \sum_{i, j=1}^{K}(-1)^{i+j} g\left(x_{i}-x_{j}\right) \cos \phi_{i} \cos \phi_{j}+\beta \sum_{j=1}^{K}(-1)^{j} \sin \phi_{j} .
$$

We proceed to compute the derivatives of $E$. Denote $\frac{\partial E}{\partial x_{k}}$ by $E_{k}$.

$$
E_{k}=\beta^{2} \sum_{j \neq k}(-1)^{k+j} g^{\prime}\left(x_{k}-x_{j}\right) \cos \phi_{j} \cos \phi_{k}+\frac{\beta^{2}}{2} \sum_{i, j=1}^{K} g\left(x_{i}-x_{j}\right)\left(\cos \phi_{i} \cos \phi_{j}\right)_{k}
$$




$$
+\beta \sum_{j=1}^{K}(-1)^{j} \cos \phi_{j} \phi_{j, k}
$$

If we differentiate (3.4) with respect to $x_{k}$, then

$$
\phi_{j, k}-(\bar{\phi})_{k}=\beta \sum_{i=1}^{K}(-1)^{i} g\left(x_{i}-x_{j}\right) \sin \phi_{i} \phi_{i, k}-\beta\left\{\begin{array}{ll}
(-1)^{k} g^{\prime}\left(x_{k}-x_{j}\right) \cos \phi_{k} & \text { if } k \neq j \\
\sum_{i \neq k}(-1)^{i} g^{\prime}\left(x_{k}-x_{i}\right) \cos \phi_{i} & \text { if } k=j
\end{array} .\right.
$$

$(\bar{\phi})_{k}$ is the derivative of $\bar{\phi}$ with respect to $x_{k}$. We multiply $(3.7)$ by $(-1)^{j} \cos \phi_{j}$ and sum over $j$. Using (3.5) we discover

$$
\begin{aligned}
\sum_{j}(-1)^{j} \cos \phi_{j} \phi_{j, k} \\
=\quad \beta \sum_{i, j=1}^{K}(-1)^{i+j} g\left(x_{i}-x_{j}\right) \sin \phi_{i} \cos \phi_{j} \phi_{i, k}-\beta \sum_{j \neq k}(-1)^{k+j} g^{\prime}\left(x_{k}-x_{j}\right) \cos \phi_{k} \cos \phi_{j} \\
\quad-\beta \sum_{i \neq k}(-1)^{i+k} g^{\prime}\left(x_{k}-x_{i}\right) \cos \phi_{i} \cos \phi_{k} \\
=\beta \sum_{i, j=1}^{K}(-1)^{i+j} g\left(x_{i}-x_{j}\right) \sin \phi_{i} \cos \phi_{j} \phi_{i, k}-2 \beta \sum_{j \neq k}(-1)^{k+j} g^{\prime}\left(x_{k}-x_{j}\right) \cos \phi_{k} \cos \phi_{j} .
\end{aligned}
$$

After we substitute the last quantity to $E_{k}$, it is simplified to

$$
E_{k}=-\beta^{2} \sum_{j \neq k}(-1)^{k+j} g^{\prime}\left(x_{k}-x_{j}\right) \cos \phi_{j} \cos \phi_{k} .
$$

In this paper we are interested in the soliton-stripe pattern with $K$ domain walls periodically placed. They are the function $\psi^{0}$, defined in (1.15), and its translates. $\psi^{0}$ corresponds to the vector $\left(x_{1}^{0}, x_{2}^{0}, \ldots, x_{K}^{0}\right)$ where $x_{j}^{0}=\frac{1}{2 K}+\frac{j-1}{K}, j=1,2, \ldots, K$. If $\left(x_{1}, \ldots, x_{K}\right)=\left(x_{1}^{0}, \ldots, x_{K}^{0}\right)$,

$$
\phi_{j}=\phi_{j}^{0}=(-1)^{j+1} \xi .
$$

Lemma $3.1\left(x_{1}^{0}, \ldots, x_{K}^{0}\right)$ and its translates are critical points of $E$.

Proof. This lemma simply restates the fact that $\phi^{0}$ is a solution of (1.14) when $\psi=\psi^{0}$. Here we give a proof. At $\left(x_{1}^{0}, \ldots, x_{K}^{0}\right)$ or any of its translates

$$
\begin{aligned}
E_{k}\left(x_{1}^{0}, \ldots, x_{K}^{0}\right) & =-\beta^{2}(\cos \xi)^{2} \sum_{j \neq k}(-1)^{k+j} g^{\prime}\left(x_{k}^{0}-x_{j}^{0}\right) \\
& = \pm \beta^{2}(\cos \xi)^{2}\left(-g^{\prime}\left(\frac{1}{K}\right)+g^{\prime}\left(\frac{2}{K}\right)-\ldots-g^{\prime}\left(\frac{K-1}{K}\right)\right) \\
& = \pm \beta^{2}(\cos \xi)^{2}\left(-\left(\frac{1}{K}-\frac{1}{2}\right)+\left(\frac{2}{K}-\frac{1}{2}\right)-\ldots-\left(\frac{K-1}{K}-\frac{1}{2}\right)\right)=0 .
\end{aligned}
$$


Next we calculate the second derivatives of $E$. Denote $\frac{\partial^{2} E}{\partial x_{k} \partial x_{l}}$ by $E_{k l}$. We separate the two cases $k \neq l$ and $k=l$. When $k \neq l$,

$$
\begin{aligned}
E_{k l}= & \beta^{2}(-1)^{k+l} g^{\prime \prime}\left(x_{k}-x_{l}\right) \cos \phi_{k} \cos \phi_{l} \\
& +\beta^{2} \sum_{j \neq k}(-1)^{k+j} g^{\prime}\left(x_{k}-x_{j}\right)\left(\sin \phi_{k} \cos \phi_{j} \phi_{k, l}+\cos \phi_{k} \sin \phi_{j} \phi_{j, l}\right) \\
= & \beta^{2}(-1)^{k+l} \cos \phi_{k} \cos \phi_{l}+\beta^{2} \sum_{j \neq k}(-1)^{k+j} g^{\prime}\left(x_{k}-x_{j}\right)\left(\sin \phi_{k} \cos \phi_{j} \phi_{k, l}+\cos \phi_{k} \sin \phi_{j} \phi_{j, l}\right)
\end{aligned}
$$

At $x_{j}=x_{j}^{0}$,

$$
\begin{aligned}
& E_{k l}\left(x_{1}^{0}, \ldots, x_{K}^{0}\right) \\
& \quad=\beta^{2}(-1)^{k+l}(\cos \xi)^{2}-\beta^{2} \sin \xi \cos \xi \sum_{j \neq k}(-1)^{k+j} g^{\prime}\left(x_{k}^{0}-x_{j}^{0}\right)\left((-1)^{k} \phi_{k, l}+(-1)^{l} \phi_{j, l}\right) \\
& \quad=\beta^{2}(-1)^{k+l}(\cos \xi)^{2}-\beta^{2} \sin \xi \cos \xi \sum_{j \neq k}(-1)^{k} g^{\prime}\left(x_{k}^{0}-x_{j}^{0}\right) \phi_{j, l}
\end{aligned}
$$

Note that we have used the fact that

$$
\sum_{j \neq k}(-1)^{j} g^{\prime}\left(x_{k}^{0}-x_{j}^{0}\right)=0
$$

as in the proof of Lemma 3.1. If $k=l$,

$$
\begin{aligned}
E_{k k}= & -\beta^{2} \sum_{j \neq k}(-1)^{k+j} g^{\prime \prime}\left(x_{k}-x_{j}\right) \cos \phi_{j} \cos \phi_{k} \\
& +\beta^{2} \sum_{j \neq k}(-1)^{k+j} g^{\prime}\left(x_{k}-x_{j}\right)\left(\sin \phi_{k} \cos \phi_{j} \phi_{k, k}+\cos \phi_{k} \sin \phi_{j} \phi_{j, k}\right)
\end{aligned}
$$

At $\left(x_{1}^{0}, \ldots, x_{K}^{0}\right)$,

$$
E_{k k}\left(x_{1}^{0}, \ldots, x_{K}^{0}\right)=\beta^{2}(\cos \xi)^{2}-\beta^{2} \sin \xi \cos \xi \sum_{j \neq k}(-1)^{k} g^{\prime}\left(x_{k}^{0}-x_{j}^{0}\right) \phi_{j, k}
$$

We write (3.10) and (3.12) in a more compact way. Define $K$ by $K$ matrices $H, F$ and $\Phi^{\prime}$ by

$$
H_{k l}=\left\{\begin{array}{ll}
(-1)^{k} g^{\prime}\left(x_{k}^{0}-x_{l}^{0}\right) & \text { if } k \neq l \\
0 & \text { if } k=l
\end{array}, F_{k l}=(-1)^{k+l}, \Phi_{k l}^{\prime}=\frac{\partial \phi_{k}}{\partial x_{l}}\left(x_{1}^{0}, \ldots, x_{K}^{0}\right) .\right.
$$

Therefore

$$
E^{\prime \prime}\left(x_{1}^{0}, \ldots, x_{K}^{0}\right)=\beta^{2}(\cos \xi)^{2} F-\beta^{2}(\sin \xi \cos \xi) H \Phi^{\prime} .
$$

It remains to compute $\Phi^{\prime}$. Define a $K$ by $K$ matrix $G$ by $G_{k l}=g\left(x_{k}^{0}-x_{l}^{0}\right)$. At $\left(x_{1}^{0}, \ldots, x_{K}^{0}\right)$ we find from (3.7) that

$$
\Phi_{j k}^{\prime}-(\bar{\phi})_{k}\left(x_{1}^{0}, \ldots, x_{K}^{0}\right)=-\beta \sin \xi \sum_{i=1}^{K} G_{j i} \Phi_{i k}^{\prime}-\beta \cos \xi H_{k j}
$$


By differentiating (3.5) we deduce

$$
\sum_{j=1}^{K}(-1)^{j} \sin \phi_{j} \phi_{j, k}=0
$$

so at $\left(x_{1}^{0}, \ldots, x_{K}^{0}\right)$

$$
\sum_{j=1}^{K} \Phi_{j k}^{\prime}=0
$$

We sum (3.14) over $j$ and by (3.15)

$$
-K(\bar{\phi})_{k}\left(x_{1}^{0}, \ldots, x_{K}^{0}\right)=-\beta \sin \xi \sum_{j, i=1}^{K} G_{j i} \Phi_{i k}^{\prime}-\beta \cos \xi \sum_{j=1}^{K} H_{k j}
$$

The right side of (3.16) is 0 since

$$
\sum_{j, i=1}^{K} G_{j i} \Phi_{i k}^{\prime}=\sum_{i=1}^{K} \Phi_{i k}^{\prime} \sum_{j=1}^{K} G_{j i}=\left(\sum_{i=1}^{K} \Phi_{i k}^{\prime}\right)\left(\sum_{j=1}^{K} g\left(\frac{j-1}{K}\right)\right)=\left(\sum_{i=1}^{K} \Phi_{i k}^{\prime}\right) \frac{1}{12 K}=0
$$

and

$$
\sum_{j=1}^{K} H_{k j}=(-1)^{k} \sum_{j=1}^{K-1} g^{\prime}\left(\frac{j}{K}\right)=(-1)^{k} \sum_{j=1}^{K-1}\left(\frac{j}{K}-\frac{1}{2}\right)=0
$$

Therefore

$$
(\bar{\phi})_{k}\left(x_{1}^{0}, \ldots, x_{K}^{0}\right)=0
$$

and (3.14) yields

$$
\Phi^{\prime}=-\beta \cos \xi(I d+\beta \sin \xi G)^{-1} H^{T}
$$

where $I d$ is the $K$ by $K$ identity matrix. To be sure that $(I d+\beta \sin \xi G)^{-1}$ exists in the last equation we note that one eigenvalue of $G$ is $1 /(12 K)$ corresponding to the eigenvector $(1,1, \ldots, 1)$ as shown in (3.17). The remaining $K-1$ eigenvalues are also positive. See (3.24).

Substituting (3.19) back to (3.13) we obtain

$$
E^{\prime \prime}\left(x_{1}^{0}, \ldots, x_{K}^{0}\right)=\beta^{2}(\cos \xi)^{2} F+\beta^{3} \sin \xi(\cos \xi)^{2} H(I d+\beta \sin \xi G)^{-1} H^{T} .
$$

Lemma $3.2 E^{\prime \prime}\left(x_{1}^{0}, \ldots, x_{K}^{0}\right)$ has one eigenvalue equal to 0 whose eigenvector is $(1,1, \ldots, 1)^{T}$, which corresponds to the translation action. The remaining $K-1$ eigenvalues are all positive.

Proof. If $q=(1,1, \ldots, 1)^{T}$, then clearly $F q=\overrightarrow{0}$ and $H^{T} q=\overrightarrow{0}$ by $(3.11)$. Hence

$$
0,(1,1, \ldots, 1)^{T}
$$

form an eigenpair.

Another eigenvector is $q=(-1,1,-1,1, \ldots,-1,1)^{T}$. Clearly $F q=K q$ and the $k$-th element of $H^{T} q$ is

$$
\sum_{j=1}^{K} H_{j k}(-1)^{j}=\sum_{j=1}^{K-1} g^{\prime}\left(\frac{j}{K}\right)=0
$$


as in (3.18). Hence $E^{\prime \prime}\left(x_{1}^{0}, \ldots, x_{K}^{0}\right) q=\beta^{2}(\cos \xi)^{2} K q$ and we have the second eigenpair

$$
\beta^{2}(\cos \xi)^{2} K, \quad(-1,1, \ldots,-1,1)^{T} .
$$

To find the remaining $K-2$ eigenpairs, for each $n=1,2, \ldots, K-1, n \neq K / 2$, we let $\theta=2 n \pi / K$ and $\zeta=e^{\sqrt{-1} \theta}$. Define $q_{\zeta}=\left(\zeta, \zeta^{2}, \zeta^{3}, \ldots, \zeta^{K}\right)^{T}$. We proceed to show that $q_{\zeta}$ is an eigenvector. It is easy to see that $F q_{\zeta}=\overrightarrow{0}$. Regarding the second part in (3.20), we need the following three identities.

$$
\sum_{j=1}^{K} \zeta^{j}=0, \quad \sum_{j=1}^{K} j \zeta^{j}=-\frac{K \zeta}{1-\zeta}, \quad \sum_{j=1}^{K} j^{2} \zeta^{j}=-\frac{K^{2} \zeta}{1-\zeta}-\frac{2 K \zeta}{(1-\zeta)^{2}} .
$$

First we compute $G q_{\zeta}$. The $k$-th entry of $G q_{\zeta}$ is

$$
\begin{aligned}
& \sum_{j=1}^{K} g\left(\frac{k-j}{K}\right) \zeta^{j} \\
& \quad=\zeta^{k} \sum_{j=1}^{K} g\left(\frac{j-k}{K}\right) \zeta^{j-k}=\zeta^{k} \sum_{j=1}^{K} g\left(\frac{j}{K}\right) \zeta^{j}=\zeta^{k} \sum_{j=1}^{K}\left(\frac{1}{2}\left(\frac{j}{K}\right)^{2}-\frac{1}{2} \frac{j}{K}+\frac{1}{12}\right) \zeta^{j} \\
& \quad=\zeta^{k}\left(\frac{1}{2 K^{2}}\left(-\frac{K^{2} \zeta}{1-\zeta}-\frac{2 K \zeta}{(1-\zeta)^{2}}\right)-\frac{1}{2 K}\left(-\frac{K \zeta}{1-\zeta}\right)\right)=-\frac{\zeta}{K(1-\zeta)} \zeta^{k}=\frac{1}{2 K(1-\cos \theta)} \zeta^{k} .
\end{aligned}
$$

Therefore

$$
G q_{\zeta}=\frac{1}{2 K(1-\cos \theta)} q_{\zeta}
$$

Hence $q_{\zeta}$ is an eigenvector of $G$. Next we compute $H^{T} q_{\zeta}$. The $k$-th entry of $H^{T} q_{\zeta}$ is

$$
\begin{aligned}
& \sum_{j \neq k}(-1)^{j} g^{\prime}\left(\frac{j-k}{K}\right) \zeta^{j} \\
& \quad=(-\zeta)^{k} \sum_{j \neq k} g^{\prime}\left(\frac{j-k}{K}\right) \zeta^{j-k}=(-\zeta)^{k} \sum_{j=1}^{K-1} g^{\prime}\left(\frac{j}{K}\right)(-\zeta)^{j}=(-\zeta)^{k} \sum_{j=1}^{K-1}\left(\frac{j}{K}-\frac{1}{2}\right)(-\zeta)^{j} \\
& \quad=(-\zeta)^{k}\left(\sum_{j=1}^{K}\left(\frac{j}{K}-\frac{1}{2}\right)(-\zeta)^{j}-\left(\frac{K}{K}-\frac{1}{2}\right)(-\zeta)^{K}\right)=(-\zeta)^{k}\left(\frac{1}{K}\left(-\frac{K(-\zeta)}{1+\zeta}\right)-\frac{1}{2}\right) \\
& \quad=\frac{\zeta-1}{2(1+\zeta)}(-\zeta)^{k}=\frac{\sqrt{-1} \sin \theta}{2(1+\cos \theta)}(-\zeta)^{k} .
\end{aligned}
$$

$q_{\zeta}$ is not an eigenvector of $H^{T}$, but

$$
H^{T} q_{\zeta}=\frac{\sqrt{-1} \sin \theta}{2(1+\cos \theta)} q_{-\zeta} .
$$

In other words $H^{T}$ sends $q_{\zeta}$ to a scalar multiple of $q_{-\zeta}$ which is made from a different angle $\theta+\pi$. Combining (3.25) and (3.24) we obtain

$$
(I d+\beta \sin \xi G)^{-1} H^{T} q_{\zeta}=\left(1+\frac{\beta \sin \xi}{2 K(1-\cos (\theta+\pi))}\right)^{-1}\left(\frac{\sqrt{-1} \sin \theta}{2(1+\cos \theta)}\right) q_{-\zeta} .
$$


Finally we compute $H q_{-\zeta}$. The $k$-th entry of $H q_{-\zeta}$ is

$$
\begin{aligned}
& \sum_{j \neq k}(-1)^{k} g^{\prime}\left(\frac{k-j}{K}\right)(-\zeta)^{j} \\
& =\zeta^{k} \sum_{j \neq k} g^{\prime}\left(\frac{k-j}{K}\right)\left(-\frac{1}{\zeta}\right)^{k-j}=\zeta^{k} \sum_{j=1}^{K-1} g^{\prime}\left(\frac{j}{K}\right)\left(-\frac{1}{\zeta}\right)^{j}=\zeta^{k} \sum_{j=1}^{K-1}\left(\frac{j}{K}-\frac{1}{2}\right)\left(-\frac{1}{\zeta}\right)^{j} \\
& =\zeta^{k}\left(\sum_{j=1}^{K}\left(\frac{j}{K}-\frac{1}{2}\right)\left(-\frac{1}{\zeta}\right)^{j}-\left(\frac{K}{K}-\frac{1}{2}\right)\left(-\frac{1}{\zeta}\right)^{K}\right)=\zeta^{k}\left(\frac{1}{K}\left(-\frac{K(-1 / \zeta)}{1+1 / \zeta}\right)-\frac{1}{2}\right) \\
& =\frac{1-\zeta}{2(1+\zeta)} \zeta^{k}=-\frac{\sqrt{-1} \sin \theta}{2(1+\cos \theta)} \zeta^{k} .
\end{aligned}
$$

So $H$ transforms $q_{-\zeta}$ back to a scalar multiple of $q_{\zeta}$, i.e.

$$
H q_{-\zeta}=-\frac{\sqrt{-1} \sin \theta}{2(1+\cos \theta)} q_{\zeta}
$$

Together with (3.26) and the fact $F q_{\zeta}=\overrightarrow{0}$ we deduce

$$
\begin{aligned}
E^{\prime \prime}\left(x_{1}^{0}, \ldots, x_{K}^{0}\right) q_{\zeta} & =\beta^{3} \sin \xi(\cos \xi)^{2} \frac{-\sqrt{-1} \sin \theta}{2(1+\cos \theta)}\left(1+\frac{\beta \sin \xi}{2 K(1+\cos \theta)}\right)^{-1} \frac{\sqrt{-1} \sin \theta}{2(1+\cos \theta)} q_{\zeta} \\
& =\frac{\beta^{3} \sin \xi(\cos \xi)^{2}(1-\cos \theta) K}{4 K(1+\cos \theta)+2 \beta \sin \xi} q_{\zeta}
\end{aligned}
$$

We thus find $K-2$ eigenpairs

$$
\frac{\beta^{3} \sin \xi(\cos \xi)^{2}(1-\cos \theta) K}{4 K(1+\cos \theta)+2 \beta \sin \xi}, q_{\zeta}
$$

where $\theta=\frac{2 \pi}{K}, \frac{4 \pi}{K}, \ldots, \frac{(K-2) \pi}{K}, \frac{(K+2) \pi}{K}, \ldots, \frac{(2 K-2) \pi}{K}$. The eigenvalues in (3.29) are all positive since $\xi \in(0, \pi / 2)$ and $\theta \in(0,2 \pi)$. Note that if $\theta$ in (3.29) is set to be 0 or $\pi$, we recover (3.21) and (3.22) respectively.

The proof of Theorem 1.1 is complete.

\section{Closing remarks}

According to $(2.14)$ at $\left(x_{1}^{0}, \ldots, x_{K}^{0}\right)$ or any of its translates

$$
\begin{aligned}
E\left(x_{1}^{0}, \ldots, x_{K}^{0}\right) & =K \int_{0}^{1 / K} \frac{\left(\phi^{\prime}\right)^{2}}{2} d x-K \beta \sin \xi \\
& =2 K^{2} \xi^{2}-K \beta \sin \xi .
\end{aligned}
$$

For large $K$ we find the expansion

$$
\xi=\frac{\beta}{4 K}-\frac{1}{2}\left(\frac{\beta}{4 K}\right)^{3} \cdots
$$


from (1.17). (4.1) gives rise to an approximation

$$
E\left(x_{1}^{0}, \ldots, x_{K}^{0}\right) \approx-\frac{\beta^{2}}{8}+\frac{\beta^{4}}{384 K^{2}} .
$$

Therefore for large $K$ we find

$$
J\left(\psi_{0}\right)+\frac{\gamma \beta^{2}}{8} \approx \tau K+\frac{\gamma \beta^{4}}{K^{2}}=\tau K+\frac{C L^{3}}{K^{2}} .
$$

In the last expression we have returned to the original parameters so that $C$ depends on $\kappa, t, s$, $\tilde{K}$, and $\lambda$, but not on $L$. This asymptotic formula that leads to the optimal spacing shows up in many other physical systems, including di- and tri-block copolymers [18, 23], and the Seul-Andelman membrane problem $[24,28]$. It is minimized at $K=\left(\frac{2 C}{\tau}\right)^{1 / 3} L$. Even though the optimal spacing

$$
\frac{L}{K}=\left(\frac{\tau}{2 C}\right)^{1 / 3}
$$

is only an approximation here, it is actually the exact value in the thermodynamic limit.

Another important formula that leads to optimal spacing is

$$
\tau K+\frac{C L^{2}}{K}
$$

which is minimized at $K=\left(\frac{C}{\tau}\right)^{1 / 2} L$. The difference between the exponents $1 / 3$ and $1 / 2$ may be significant. In [25] we showed that (4.4) appears in a charged Langmuir monolayer problem proposed by Andelman et al [1]. It is also found in the study of domain structures of superconductors in the intermediate state [29], and ferromagnets [13].

It is natural to extend the one-dimensional soliton-stripe solutions trivially to a two-dimension square. They continue to be solutions of the Euler-Lagrange equations of (1.5). Whether they are still local minimizers in two-dimensions is not clear. The techniques we developed for the diblock copolymer problem in $[21,26]$ should help in a future study.

Acknowledgments. The support from Institute of Mathematical Sciences at Chinese University of Hong Kong is very much appreciated.

\section{References}

[1] D. Andelman, F. Broçhard, and J.-F. Joanny. Phase transitions in Langmuir monolayers of polar molecules. J. Chem. Phys., 86(6):3673-3681, 1987.

[2] A. Chmaj and X. Ren. Multiple layered solutions of the nonlocal bistable equation. Physica D, 147(1-2):135-154, 2000.

[3] R. Choksi. Scaling laws in microphase separation of diblock copolymers. J. Nonlinear Sci., 11:223-236, 2001.

[4] R. Choksi and X. Ren. On the derivation of a density functional theory for microphase separation of diblock copolymers. J. Statist. Phys., 113(1\&2):151-176, 2003. 
[5] G. Dal Maso. Introduction to Gamma-Convergence. Progress in Nonlinear Differential Equations and Their Applications, Vol 8. Birkhäuser, Boston, 1992.

[6] P.G. De Gennes. An analogy between superconductivity and smectics a. Solid State Commun., 10:753-7576, 1972.

[7] P.G. De Gennes and J. Prost. The Physics of Liquid Crystals. Oxford University Press, second edition, 1995.

[8] E. De Giorgi. Sulla convergenza di alcune successioni di integrali del tipo della'area. Rendiconti di Matematica, 8:277-294, 1975.

[9] L.C. Evans and R.F. Gariepy. Measure Theory and Fine Properties of Functions. CRC Press, Boca Raton, New York, London, Tokyo, 1992.

[10] P.C. Fife and D. Hilhorst. The Nishiura-Ohnishi free boundary problem in the 1D case. SIAM J. Math. Anal., 33(3):589-606, 2001.

[11] M. Henry. Singular limit of a fourth order problem arising in the micro-phase separation of diblock copolymers. Adv. Differential Equations, 6(9):1049-1114, 2001.

[12] R. Kohn and P. Sternberg. Local minimisers and singular perturbations. Proc. Royal Soc. Edin., 111A:69-84, 1989.

[13] L.D. Landau, E.M. Lifshitz, and L.P. Pitaevskii. Electrodynamics of Continuous Media, Course of Theoretical Physics, Volume 8. Butterworth-Heinemann, second edition, 1984.

[14] L. Modica. The gradient theory of phase transitions and the minimal interface criterion. Arch. Rat. Mech. Anal., 98:357-383, 1987.

[15] Y. Nishiura and I. Ohnishi. Some mathematical aspects of the microphase separation in diblock copolymers. Physica D, 84:31-39, 1995.

[16] I. Ohnishi, Y. Nishiura, M. Imai, and Y. Matsushita. Analytical solutions describing the phase separation driven by a free energy functional containing a long-range interaction term. Chaos, 9(2):329-341, 1999.

[17] X. Ren and L. Truskinovsky. Finite scale microstructures in nonlocal elasticity. In recognition of the sixtieth birthday of Roger L. Fosdick (Blacksburg, VA, 1999). J. Elasticity, 59(1-3):319-355, 2000.

[18] X. Ren and J. Wei. On the multiplicity of solutions of two nonlocal variational problems. SIAM J. Math. Anal., 31(4):909-924, 2000.

[19] X. Ren and J. Wei. Concentrically layered energy equilibria of the di-block copolymer problem. European J. Appl. Math., 13(5):479-496, 2002.

[20] X. Ren and J. Wei. On energy minimizers of the di-block copolymer problem. Interfaces Free Bound., 5:193-238, 2003.

[21] X. Ren and J. Wei. On the spectra of 3-D lamellar solutions of the diblock copolymer problem. SIAM J. Math. Anal., 35(1):1-32, 2003. 
[22] X. Ren and J. Wei. Triblock copolymer theory: Free energy, disordered phase and weak segregation. Physica D, 178:103-117, 2003.

[23] X. Ren and J. Wei. Triblock copolymer theory: Ordered ABC lamellar phase. J. Nonlinear Sci., 45(2):175-208, 2003.

[24] X. Ren and J. Wei. The soliton-stripe pattern in the Seul-Andelman membrane. Phsica D, in press.

[25] X. Ren and J. Wei. Soliton-stripe patterns in charged Langmuir monolayers. J. Nonlinear Sci., in press.

[26] X. Ren and J. Wei. Wriggled lamellar solutions and their stability in the diblock copolymer problem. preprint.

[27] J.V. Selinger, Z.-G. Wang, R.F. Bruinsma, and C.M. Knobler. Chiral symmetry breaking in Langmuir monolayers and smectic films. Phys. Rev. Lett., 70(8):1139-1142, 1993.

[28] M. Seul and D. Andelman. Domain shapes and patterns: The phenomenology of modulated phases. Science, 267:476-483, 1995.

[29] M. Tinkham. Introduction to Superconductivity. McGraw-Hill, second edition, 1995. 